\title{
CONTINUOUS IMAGES OF BOREL SETS
}

\section{MAURICE SION}

1. Introduction. It is well known that in a complete separable metric space every Borel set is the one-to-one continuous image of a $g_{\delta}$ in some other such space and that the countable-to-one continuous image of a Borel set is Borel.

In a general topological space, if $K$ denotes the family of compact sets, G. Choquet [1] has pointed out that the proper families to consider in this context are Borelian $K$ instead of Borel $K$ and $K_{\sigma \varepsilon}$ instead of $g_{8}$ (see $\$ 2$ for definitions). Let a space have property $I$ if it is Hausdorff and the difference of two compact sets is a $K_{\sigma}$. It has been shown by Choquet [1] that if $Y$ has property I then every Borelian $K$ set in $Y$ is the one-to-one continuous image of a $K_{\sigma \delta}$ in some compact Hausdorff $X$. On the other hand, in a previous paper [2] we proved that if $X$ has property $I$ then the countable-to-one continuous image of a $K_{\sigma \delta}$ in $X$ to a compact Hausdorff space $Y$ is Borelian $K$ in $Y$.

In this paper we complete the picture. We first show that the difference of two compact sets is a $K_{\sigma}$ iff it is analytic and conclude that a space has property $I$ iff it is Hausdorff and Borel $K=$ Borelian $K$, thereby answering a question raised by Choquet $[1, \mathrm{p} .139]$. We then prove that if $X$ has property $I$ then every Borel $K$ set in $X$ is the one-to-one continuous image of a $K_{\sigma \delta}$ in some $Y$, where $Y$ also has property I. Making use of our previous result, we conclude that the countable-to-one continuous image of a Borel $K$ set in $X$ to a compact Hausdorff space $Y$ is Borelian $K$ and it too has property $\mathrm{I}$.

We are unable to determine whether the condition that $X$ have property I can be eliminated from the hypotheses, i.e., whether in any compact Hausdorff space $X$, every Borelian $K$ set is the one-to-one continuous image of a $K_{o b}$ in some other compact Hausdorff space and whether the countable-to-one (or even one-to-one) image of a Borelian $K$ (or even a $K_{\sigma 8}$ ) set in $X$ into a compact Hausdorff space is also Borelian $K$.

\section{Notation and basic definitions.}

2.1. $\omega$ denotes the set of all non-negative integers.

2.2. $K(X)$ is the family of all compact sets in $X$.

2.3. $K_{\sigma}(X)=\left\{A: A=\bigcup_{i \in \omega} B_{i}\right.$ for some sequence $B$ with $B_{i} \in K(X)$ for $i \in \omega\}$.

2.4. $K_{o \delta}(X)=\left\{A: A=\bigcap_{i \in \omega} B_{i}\right.$ for some sequence $B$ with $B_{i} \in K_{\sigma}(X)$ for $i \in \omega\}$.

Received by the editors June 3, 1960. 
2.5. Borel $K(X)$ is the smallest family $H$ such that $K(X) \subset H$ and if $A_{i} \in H$, for $i \in \omega$, then $\bigcup_{i \in \omega} A_{i} \in H$ and $A_{0}-A_{1} \in H$.

2.6. Borelian $K(X)$ is the smallest family $H$ such that $K(X) \subset H$ and if $A_{i} \in H$, for $i \in \omega$, then $\bigcup_{i \in \omega} A_{i} \in H$ and $\bigcap_{i \in \omega} A_{i} \in H$.

2.7. $A$ is analytic in $X$ iff $A$ is the continuous image of a $K_{o \delta}\left(X^{\prime}\right)$ for some Hausdorff space $X^{\prime}$.

2.8. $X$ has property I iff $X$ is Hausdorff and, for every $A$ and $B$ in $K(X), A-B \in K_{\sigma}(X)$.

2.9. $X$ has a countable compact base iff $X$ is Hausdorff and there exists a sequence $C$ with $C_{i} \in K(X)$, for $i \in \omega$, such that if $A$ is open in $X$ and $x \in A$ then, for some $i \in \omega, x \in C_{i} \subset A$.

2.10. $\prod_{i \in \omega} Y_{i}$ denotes the cartesian product of the $Y_{i}$, for $i \in \omega$.

2.11. The union topology for $\bigcup_{i \in \omega} Y_{i}$, where $Y_{i} \cap Y_{j}=0$ for $i \neq j$, is the topology in which $A$ is open iff $A=\bigcup_{i \in \omega} \alpha_{i}$ where $\alpha_{i}$ is open in $Y_{i}$ for $i \in \omega$.

3. Property I and countable compact bases. In this section, we study conditions under which a set has property I or a countable compact base. The main results are Theorems 3.1, 3.3, 3.5. The other results are needed in the next section.

3.1. Theorem. Let $X$ be Hausdorff, $A$ and $B$ in $K(X)$. Then $A-B$ is analytic in $X$ iff $A-B \in K_{\sigma}(X)$.

Proof. If $A-B \in K_{\sigma}(X)$ then clearly $A-B$ is analytic in $X$. Suppose now that $A-B$ is analytic in $X$. Then by Theorem 2.3 in [3], $A-B$ is Lindelöf in $X$, i.e., any open covering of $A-B$ can be reduced to a countable subcovering. Let

$$
\begin{aligned}
& G=\{\beta: \beta \text { is open in } X \text { and } B \subset \beta\}, \\
& F=\{\alpha: \alpha=X-\text { closure } \beta \text { for some } \beta \in G\} .
\end{aligned}
$$

Since $X$ is Hausdorff, $F$ is an open covering of $X-B$ and hence a countable subfamily $F^{\prime}$ covers $A-B$. Let $G^{\prime}$ be a countable subfamily of $G$ such that

$$
F^{\prime}=\left\{\alpha: \alpha=X-\text { closure } \beta \text { for some } \beta \in G^{\prime}\right\}
$$

and let

$$
H=\left\{\gamma: \gamma=A-\beta \text { for some } \beta \in G^{\prime}\right\} ;
$$

then $H$ is a countable family of compact sets whose union is $A-B$ so that $A-B \in K_{\sigma}(X)$.

3.2. Lemma. Borelian $K(X) \subset$ Borel $K(X)$. 
3.3. Theorem. $X$ has property I. iff $X$ is Hausdorff and Borelian $K(X)=$ Borel $K(X)$.

Proof. Suppose $X$ has property I. Let $H$ be a maximal family such that $K(X) \subset H$ and if $A$ and $B$ are in $H$ then $A$ and $A-B$ are Borelian $K(X)$. Then we easily check that $H$ is closed under countable unions and intersections so that $H=$ Borelian $K(X)$. Thus, if $A$ and $B$ are Borelian $K(X)$ then $A-B$ is also Borelian $K(X)$. Therefore Borel $K(X) \subset$ Borelian $K(X)$ and in view of 3.2, Borel $K(X)=$ Borelian $K(X)$.

Next, suppose $X$ is Hausdorff and Borelian $K(X)=$ Borel $K(X)$. If $A$ and $B$ are in $K(X)$ then $A-B$ is Borelian $K(X)$ and hence (see e.g. $[1$, p. 142]) $A-B$ is analytic in $X$. Therefore, by $3.1, A-B$ $\in K_{\sigma}(X)$.

3.4. Lemma. $X$ has property I iff $X$ is Hausdorff and for every $A \in K(X)$ and $B$ open in $X$ we have $A \cap B \in K_{\sigma}(X)$.

3.5. TheOREM. If $Y$ has a countable compact base and $X$ has property I then $X \times Y$ has property I.

Proof. Let $C$ be a sequence of compact sets in $Y$ such that if $U$ is open in $Y$ and $y \in U$ then, for some $i \in \omega, y \in C_{i} \subset U$. Suppose $A$ is compact and $B$ is open in $X \times Y$. Let, for each $i \in \omega$,

$$
\beta_{i}=\left\{x:\{x\} \times C_{i} \subset B\right\} .
$$

Then the $\beta_{i}$ are open in $X$. Moreover,

$$
B=\bigcup_{i \in \omega}\left(\beta_{i} \times C_{i}\right)
$$

for, if $(x, y) \in B$ then, for some $i \in \omega$,

$$
y \in C_{i} \subset\{z:(x, z) \in B\}
$$

and hence $x \in \beta_{i}$ and $(x, y) \in \beta_{i} \times C_{i}$. Let $\alpha$ be the projection of $A$ onto $X$. Then $\alpha$ is compact in $X$ and $\alpha \cap \beta_{i} \in K_{\sigma}(X)$ and hence

$$
\left(\alpha \cap \beta_{i}\right) \times C_{i} \in K_{\sigma}(X \times Y) .
$$

Since

$$
A \cap B=\bigcup_{i \in \omega}\left(A \cap\left(\beta_{i} \times C_{i}\right)\right)=\bigcup_{i \in \omega}\left(A \cap\left(\left(\alpha \cap \beta_{i}\right) \times C_{i}\right)\right)
$$

we see that $A \cap B \in K_{\sigma}(X \times Y)$. Therefore $X \times Y$ has property I.

3.5a. Corollary. If $Y$ is a metric space and $X$ has property I then $X \times Y$ has property $\mathrm{I}$. 
Proof. If $A \in K(X \times Y)$, let $B$ be the projection of $A$ onto $Y$. Then $B$ is a compact metric space and hence has a countable compact base and $A \subset X \times B$ so that we may apply 3.5.

3.6. Theorem. If $X$ has property $\mathrm{I}, C \in K_{\sigma \delta}(X), f$ is continuous on $C$, and $f(C)$ is Hausdorff then $f(C)$ has property $\mathrm{I}$.

Proof. Let $A$ be compact and $B$ open in $f(C)$. Then $f^{-1}(A)$ is closed in $C$ and hence $f^{-1}(A) \in K_{\text {os }}(X)$. Since $f^{-1}(B)$ is open in $C$ and $X$ has property $\mathrm{I}$, we conclude

$$
f^{-1}(A \cap B)=f^{-1}(A) \cap f^{-1}(B) \in K_{\sigma \delta}(X) .
$$

Thus, $A \cap B$ is the continuous image of a $K_{\sigma \delta}(X)$, i.e., $A \cap B$ is analytic in $f(C)$, and hence, by 3.1, $A \cap B \in K_{\sigma}(f(C))$.

3.7. Theorem. Let $f$ be continuous and one-to-one on $C$ and $f(C)$ have property I. Then $C$ has property I.

Proof. Clearly $C$ must be Hausdorff. If $A$ and $B$ are compact in $C$, then $f(A)$ and $f(B)$ are compact in $f(C)$ and hence $f(A)-f(B)$ $\in K_{\sigma}(f(C))$. Since $f$ is one-to-one, we have

$$
A-B=A \cap f^{-1}(f(A)-f(B)) \in K_{\sigma}(C) .
$$

3.8. Lemma. If, for each $i \in \omega, Y_{i}$ is compact and has a countable compact base, then $\prod_{i \in \omega} Y_{i}$ is compact and has a countable compact base in the product topology.

3.9. Lemma. If, for each $i \in \omega, Y_{i}$ is compact and has a countable compact base and $Y_{i} \cap Y_{j}=0$ for $i \neq j$ then $\bigcup_{i \in \omega} Y_{i}$ is locally compact and has a countable compact base in the union topology.

3.10. LemMa. If $Y$ is locally compact and has a countable compact base then its one point compactification has a countable compact base.

4. Continuous images of Borel sets. In this section we study oneto-one projections of $K_{\sigma \delta}$ sets and continuous countable-to-one images of Borel sets. The main results are Theorems 4.6 and 4.7.

4.1. Lemma. If, for each $i \in \omega, Y_{i}$ is compact and $A_{i}$ is the one-to-one projection of $a K_{o \delta}\left(X \times Y_{i}\right)$ onto $X$ then $\bigcap_{i \in \omega} A_{i}$ is the one-to-one projection of $a$

$$
K_{\sigma \delta}\left(X \times \prod_{i \in \omega} Y_{i}\right) \quad \text { onto } X
$$

Proof. For each $i \in \omega$, let $C_{i} \in K_{o \delta}\left(X \times Y_{i}\right)$ and $A_{i}$ be the one-to-one 
projection of $C_{i}$ onto $X$. For $x \in A_{i}$, let $h_{i}(x)$ be the $y$ such that $(x, y) \in C_{i}, Z=\prod_{i \in \omega} Y_{i}$, and

$$
F_{i}=\left\{(x, y): x \in A_{i}, y \in Z \text { and } y_{i}=h_{i}(x)\right\} .
$$

Then $F_{i}$ is homeomorphic to $C_{i} \times \prod_{j \in(\omega-(i))} \quad Y_{j}$ so that $F_{i} \in$ $K_{\text {os }}(X \times Z)$. Let

$$
D=\bigcap_{i \in \omega} F_{i} .
$$

Then $D \in K_{\sigma \delta}(X \times Z)$ and $(x, y) \in D$ iff $x \in \bigcap_{i \in \omega} A_{i}$ and $y_{i}=h_{i}(x)$ for all $i \in \omega$. Thus, if $(x, y) \in D$ and $\left(x, y^{\prime}\right) \in D$ then $y_{i}=h_{i}(x)=y_{i}^{\prime}$ for all $i \in \omega$ so that $y=y^{\prime}$ and $\bigcap_{i \in \omega} A_{i}$ is the one-to-one projection of $D$ onto $X$.

4.2. Lemma. If, for each $i \in \omega, A_{i}$ is the one-to-one projection of a $K_{o \delta}\left(X \times Y_{i}\right)$ onto $X, A_{i} \cap A_{j}=0=Y_{i} \cap Y_{j}$ for $i \neq j$, and $Y^{\prime}=U_{i \in \omega} Y_{i}$ with the union topology then $\cup_{i \in \omega} A_{i}$ is the one-to-one projection of a $K_{\text {os }}\left(X \times Y^{\prime}\right)$ onto $X$.

Proof. Let $C_{i} \in K_{\sigma \delta}\left(X \times Y_{i}\right), A_{i}$ be the one-to-one projection of $C_{i}$ onto $X, D=\cup_{i \in \omega} C_{i}$. Then $\cup_{i \in \omega} A_{i}$ is the one-to-one projection of $D$. Moreover, $D$ is a $K_{o \delta}\left(X \times Y^{\prime}\right)$, for if

$$
C_{i}=\bigcap_{j \in \omega} B(i, j) \quad \text { with } B(i, j) \in K_{\sigma}\left(X \times Y_{i}\right)
$$

then for $i \neq i^{\prime}, B(i, j) \cap B\left(i^{\prime}, k\right)=0$ for all $j \in \omega, k \in \omega$ and hence

$$
D=\bigcup_{i \in \omega} \bigcap_{j \in \omega} B(i, j)=\bigcap_{\in \omega} \bigcup_{i \in \omega} B(i, j)
$$

and

$$
\bigcup_{i \in \omega}\left(B_{i, j}\right) \in K_{\sigma}\left(X \times Y^{\prime}\right) .
$$

4.3. Definition. $A$ is a special set of uniqueness in $X$ iff there exist $Y$ and $C$ such that $Y$ is compact and has a countable compact base and $C \in K_{\theta \delta}(X \times Y)$ and $A$ is the one-to-one projection of $C$ onto $X$.

4.4. Theorem. If for each $i \in \omega, A_{i}$ is a special set of uniqueness in $X$ then $\cap_{i \in \omega} A_{i}$ is a special set of uniqueness in $X$ and if $A_{i} \cap A_{j}=0$ for $i \neq j$ then $\cup_{i \in \omega} A_{i}$ is a special set of uniqueness in $X$.

Proof. $\bigcap_{i \in \omega} A_{i}$ is a special set of uniqueness in $X$ in view of 4.1 and 3.8. To see that if $A_{i} \cap A_{j}=0$ for $i \neq j$ then $\bigcup_{i \in \dot{\omega}} A_{i}$ is a special set of uniqueness in $X$, let $A_{i}$ be the one-to-one projection of $C_{i}$ where $C_{i} \in K_{o \delta}\left(X \times Y_{i}\right)$ and $Y_{i}$ is compact and has a countable compact 
base. We may assume that $Y_{i} \cap Y_{j}=0$ for $i \neq j$ for otherwise we may replace $Y_{i}$ by $Y_{i} \times\{i\}$. Let $Y^{\prime}=\cup_{i \in \omega} Y_{i}$ with the union topology and $Z$ be the one-point compactification of $Y^{\prime}$. Then by Lemmas 3.9 and $3.10, Z$ is compact and has a countable compact base. Moreover $K_{\sigma \delta}\left(X \times Y^{\prime}\right) \subset K_{\sigma \delta}(X \times Z)$. Hence by $4.2, \bigcup_{i \in \omega} A_{i}$ is a special set of uniqueness in $X$.

4.5. Theorem. If $X$ has property I and $A \in$ Borel $K(X)$ then $A$ is a special set of uniqueness in $X$.

Proof. If $A \in K_{\sigma \delta}(X)$ then clearly $A$ is a special set of uniqueness in $X$ since we can take $Y=\{0\}$ and $A$ is the projection of $A \times\{0\}$ $\in K_{\sigma \delta}(X \times Y)$. Let $H$ be a maximal family such that $K(X) \subset H$ and if $A$ and $B$ are in $H$ then $A$ and $A-B$ are special sets of uniqueness in $X$. We shall show that $H$ is closed under countable unions and difference of two sets so that Borel $K(X) \subset H$. Let $A_{i} \in H$, for $i \in \omega$, and

$$
S=\{A: A \text { is a special set of uniqueness in } X\} \text {. }
$$

We now check, using 4.4 :

(i) $A_{0}-A_{1} \in H$, for, $A_{0}-A_{1} \in S$ and for any $B \in H$,

$$
\left(A_{0}-A_{1}\right)-B=\left(A_{0}-A_{1}\right) \cap\left(A_{0}-B\right) \in S
$$

and

$$
B-\left(A_{0}-A_{1}\right)=\left(B-A_{0}\right) \cup\left(B \cap A_{0} \cap A_{1}\right) \in S ;
$$

(ii) $A_{0} \cup A_{1} \in H$ and hence

$$
\bigcup_{i=0}^{n} A_{i} \in H \quad \text { for } n \in \omega
$$

for,

$$
A_{0} \cup A_{1}=A_{0} \cup\left(A_{1}-A_{0}\right) \in S
$$

and for any $B \in H$,

$$
\begin{aligned}
& \left(A_{0} \cup A_{1}\right)-B=\left(A_{0}-B\right) \cup\left(\left(A_{1}-B\right) \cap\left(A_{1}-A_{0}\right)\right) \in S, \\
& B-\left(A_{0} \cup A_{1}\right)=\left(B-A_{0}\right) \cap\left(B-A_{1}\right) \in S ;
\end{aligned}
$$

(iii) $\bigcup_{i \in \omega} A_{i} \in H$, for, let

$$
A_{n}^{\prime}=A_{n}-\bigcup_{i=0}^{n-1} A_{i} .
$$

Then by (i) and (ii), $A_{n}^{\prime} \in H$ and $A_{n}^{\prime} \cap A_{k}^{\prime}=0$ for $n \neq k$. Hence 


$$
\bigcup_{i \in \omega} A_{i}=\bigcup_{n \in \omega} A_{n}^{\prime} \in S
$$

and for any $B \in H$,

$$
\begin{aligned}
& \bigcup_{i \in \omega} A_{i}-B=\bigcup_{n \in \omega}\left(A_{n}^{\prime}-B\right) \in S, \\
& B-\bigcup_{i \in \omega} A_{i}=\bigcap_{i \in \omega}\left(B-A_{i}\right) \in S .
\end{aligned}
$$

4.6. Theorem. Let $X$ have property I. Then $A$ is Borel $K(X)$ iff, for some $B \in K_{\sigma}(X), A \subset B$ and there exist $X^{\prime}, C, f$ such that $X^{\prime}$ has property $\mathrm{I}, C \in K_{\text {os }}\left(X^{\prime}\right), f$ is continuous and one-to-one on $C$ and $A=f(C)$.

Proof. If $A \in$ Borel $K(X)$ then by $4.5 A$ is the one-to-one projection of a $K_{\sigma \delta}(X \times Y)$ for some $Y$ that is compact and has a countable compact base. By 3.5, $X \times Y$ has property I. Moreover, since $X$ has property I, by 3.3 Borel $K(X)=$ Borelian $K(X)$ and hence $A \subset B$ for some $B \in K_{\sigma}(X)$.

The converse is given by Theorem 6.3 in [2] again with the help of 3.3.

4.7. Theorem. If $X$ has property $\mathrm{I}, A$ is Borel $K(X), f$ is continuous and countable-to-one on $A$ to some Hausdorff space $Y$, and $Y \in K_{*}(Y)$ then $f(A)$ is Borelian $K(Y)$ and $f(A)$ has property $\mathrm{I}$.

Proof. In view of $4.6, f(A)$ is the continuous countable-to-one image of a $K_{\sigma \delta}\left(X^{\prime}\right)$ for some $X^{\prime}$ that has property I. Hence by Corollary 6.10 in [2], $f(A)$ is Borelian $K(Y)$. By 3.6, $f(A)$ also has property I.

\section{BIBLIOGRAPHY}

1. G. Choquet, Theory of capacities, Ann. Inst. Fourier Grenoble vol. 5 (19531954) pp. 131-295.

2. M. Sion, On analytic sets in topological spaces, Trans. Amer. Math. Soc. vol. 96 (1960) pp. 341-354.

3. - Topological and measure theoretic properties of analytic sets, Proc. Amer. Math. Soc. vol. 11 (1960) pp. 769-776.

University of California, Berkeley 